\title{
Philosophical Considerations and Research Ideas About Comparing the Two Approaches: Messer's Comments Point the Way Forward
}

\author{
MICHAEL A. WESTERMAN, ${ }^{\mathrm{a}, \mathrm{c}}$ \& KENNETH L. CRITCHFIELD ${ }^{\mathrm{b}}$
}

\author{
${ }^{\mathrm{a}}$ New York University \\ b James Madison University \\ ${ }^{\mathrm{c}}$ Correspondence regarding this article should be sent to: Michael A. Westerman, Department of Psychology, New \\ York University, 6 Washington Place, Fourth Floor, New York, NY 10003, USA. \\ Email: michael.westerman@nyu.edu
}

\begin{abstract}
In his commentary, Stanley Messer (2021) posed the question of whether it is possible to evaluate the relative merits of different case formulation approaches to psychotherapy. He went on to maintain, based on the pragmatic theory of truth, that it is possible to compare different case formulation approaches, and pointed to a program of research that he and his collaborators conducted as an example for possible future research (Collins \& Messer, 1991; Holland, Roberts, \& Messer, 1998; Messer, Tishby, \& Spillman, 1992; Tishby \& Messer, 1995). In this reply, we express our appreciation for Messer's remarks, with which we agree in large measure, and attempt to highlight and build upon some of the points he made. We discuss Dewey's (1896) classic critique of the reflex arc concept to point out other ways the philosophical perspective of pragmatism supports the view that different approaches to therapy are not incommensurate. We also offer a number of suggestions for future research comparing psychotherapy based on Interpersonal Defense Theory and IRT, or any two case formulation approaches to therapy. At many points, our suggestions follow along the lines of Messer's research. We also emphasize the value of case formulation-based studies, not only with regard to research comparing approaches to treatment, but for investigating other issues about therapy as well.
\end{abstract}

Keywords: Interpersonal Defense Theory; Interpersonal Reconstructive Therapy (IRT); philosophy; pragmatism; case formulation; case study; clinical case study

In his commentary, which he described as a "meta-view," Stanley Messer (2021) has offered important considerations about our attempts to compare therapy based on Interpersonal Defense Theory (Westerman, 2021a, 2021b) with Interpersonal Reconstructive Therapy (IRT; Critchfield, Dobner-Pereira, \& Stucker, 2021a, 2021b), and he has presented valuable suggestions about how to move forward from this point. In large measure, we agree with what he has said, and we offer this reply to his commentary as an attempt to highlight certain points he made and build upon them. 
We especially appreciate Messer's commentary because it included consideration of basic philosophical matters in addition to - and linked to - suggestions about future research. In particular regarding philosophy, we agree that it is helpful to turn to pragmatism for insights about how to proceed, although what we take from pragmatism is rather different from (but not incompatible with) how Messer draws on that philosophical perspective.

We also think that Messer's remarks about research are quite helpful. We certainly agree with him that the relative merits of our two approaches to therapy can be evaluated; they are not incommensurate. Moreover, the research Messer and his colleagues conducted comparing the Mt Zion approach based on control-mastery theory and an approach based on object relations theory serves as a very helpful example of possible ways to pursue such investigations (Collins \& Messer, 1991; Holland, Roberts, \& Messer, 1998; Messer, Tishby, \& Spillman, 1992; Tishby \& Messer, 1995). One of the features of that research which we view as especially valuable is that it was based on case formulations. Another valuable feature of those studies is that they show how investigators can conduct quantitative studies comparing theoretical approaches to psychotherapy in a way that focuses intensively on a small number of cases (specifically, two cases in the research just cited).

In what follows, we begin with philosophical considerations related to Messer's remarks about three philosophical positions about truth, the correspondence theory of truth, the coherence theory of truth, and the pragmatic theory of truth. We then turn to issues regarding directions for future research, taking Messer's research as a touchstone for our remarks.

\section{PHILOSOPHICAL CONSIDERATIONS}

In his commentary, Messer maintained that the pragmatic theory of truth is the most helpful for comparing different psychotherapy approaches because, in contrast to correspondence and coherence theories of truth, it provides a basis for investigating what difference it makes if therapists proceed according to one approach as compared to another. However, questions of this sort certainly can be investigated based on correspondence theory. Indeed, the majority of researchers in psychology implicitly or explicitly are guided by the view that they are trying to learn truths about psychological phenomena, as "truth" is understood according to correspondence theory, and many of the truths they attempt to arrive at concern what will promote particular outcomes.

Probably the most common example philosophers use to explain the correspondence theory of truth involves a descriptive claim about a cat. According to correspondence theory, the statement "The cat is on the mat" is true if and only if the cat is on the mat, that is, does the statement match the way things are in the world. However, this view applies equally well to claims about causal relationships, for example, "Ingesting lead [as in lead paint] during infancy increases the likelihood of attention deficit disorder later in childhood," or, more to the point regarding present concerns, "Therapy guided by theoretical approach $\mathrm{X}$ to case formulation will lead to better outcomes than therapy guided by approach $\mathrm{Y}$ to case formulation." According to correspondence theory, those claims are true if and only if they match what happens in the world. 
Whereas it is quite clear that correspondence theory provides one possible philosophical basis for comparing the effects of two therapy approaches, it is much less clear that coherence theory provides a foundation for such efforts. Nevertheless, some proponents of coherence theory have argued that it can play that role. For example, Spence (1982), whom Messer cited, claimed that the aesthetic quality of narratives developed in therapy about links between patients' early histories and their current problems affects therapy outcomes. And according to Spence, aesthetic quality depends on how coherent those narratives are as stories and not on whether they match what actually transpired during patients' childhoods.

Whether or not it is too much of a stretch to say that coherence theory serves as a possible basis for investigating differential therapy outcomes, the correspondence theory of truth certainly does. This raises the question of whether the pragmatic theory of truth and pragmatism more generally have anything special to offer regarding the present set of articles. Here, we are in agreement with Messer, in the sense that we believe it does. However, our view of what pragmatism has to offer differs from his.

\section{Dewey's Critique of the Reflex Arc}

Dewey's (1896) classic critique of the reflex arc concept serves as an excellent basis for presenting key features of pragmatism. In that article, Dewey maintained that sensory experiences and behavioral responses are not independent building block phenomena that become linked together. Rather, they should be treated as parts of action patterns. For example, what a person hears at a particular point in time in a given situation will depend on what he or she was already engaged in doing, perhaps waiting for a friend who should be driving up to the house any minute now, or, instead, walking through the woods hoping to photograph a certain kind of bird that has a distinctive song. In such cases, prior to hearing a sound, the person will already be poised in a certain manner (e.g., tilting his or her head and/or closing his or her eyes to avoid visual distractions).

This idea about the primacy of action patterns, or what we will refer to as practical activities or practices, has a number of important implications. For one thing, Dewey argued that it moved psychology beyond subject-world dualism. In what follows, we point out several other implications.

The primacy of practices also points to the anti-foundationalist stance of pragmatism. That is, pragmatism rejects the view that we could ever arrive at final, complete, absolute accounts of the nature of things. Instead, it maintains that truths are always provisional; they can change and indeed it is likely that they will change at some later point in time. The reason for this is that practices change.

Correspondence theory also can make sense of change, whether that involves the observation that a billiard ball sitting still in one place on a pool table will start moving off in a certain direction at a certain speed when another ball hits it, or observations indicating that a star that had been moving through space in a certain manner for millions of years or longer changed trajectory and got swallowed up by a black hole. However, according to correspondence theory, 
there are laws that govern these events that tell us this is the way things work, and those laws are invariant. They provide final, absolute accounts that are grounded in the objective nature of things.

According to pragmatism the truth about how things work itself can change if and when our lives change. As Messer pointed out in his commentary, whereas correspondence theory is based on the idea that the truth about things is independent of the observer, pragmatism views truth in terms of whether beliefs work for our lives. This is the "cash value" Messer referred to quoting William James. Truths will change over time if and when our lives change because what outcomes count as having cash value will change and what promotes outcomes with cash value will also change.

Does this mean that what counts as true according to pragmatism has no grounding, as is the case for coherence theory? No, it does not. According to pragmatism, because our practical activities are primary, we cannot ground truth in the objective nature of things, that is, in what they are like in themselves independent of ourselves. However, our shared practices provide a different kind of grounding, or cornerstone, for our beliefs. Although practices change, at any point in time what they are like then is our current starting point.

We can explain this point about a different kind of grounding by offering some comments about the nature of practices. To begin with, practices include all the things we do. For example, they include growing crops for food. However, notwithstanding our use of the term "practical activities," they are not limited to things we do that are obviously useful. For example, they include staring with wonder at the stars or relaxing while lying on the beach. Also, they are neither primarily ideal (as in "ideas") in nature nor merely behaviors. Instead, they are the concretely meaningful action patterns that people engage in. As such, they include but are not equivalent to chairs and tables, ideas and theories, things we say, motor behaviors, instrumentation, institutions, and so forth. The items in this list and others as well are parts of practices. In addition, the fact that people are embodied is at least as important as, and intrinsically linked to, how we reflect on things. Also, practices are shared, sometimes by almost all human beings (e.g., how we walk) and sometimes by particular groups of people (using one's fingers to eat in some cultures or using utensils in others).

All these features of practices - but perhaps especially their concrete rather than ideal nature and the fact that they are shared - constitute a kind of ground for what counts as true, although not the absolute ground supposedly provided, according to correspondence theory, by what things are like in themselves. As a result, according to pragmatism, truth is not an arbitrary matter that individuals can choose for themselves based on their own preferences or however they happen to think about things. Similarly, for pragmatists and in contrast to the views of most 
social constructionists, truth is not an arbitrary matter of convention or of the language of any given discourse community. ${ }^{1}$

The primacy of practical activities also leads to the idea that the study of psychological phenomena is an interpretive enterprise. As just noted, this reference to interpretation does not mean that it is a matter of how a theorist or researcher chooses to think about things. Rather, the point is that meaning is an irreducible part of theorizing and research.

There are two reasons why this is the case. The first is that we have to view the phenomena we study in a way that takes concretely meaningful action patterns as our starting point. Most research in psychology attempts to avoid concerns about meaning by proceeding in the opposite direction, building up accounts of what people do starting with perceptions, cognitions, feelings, and individual behaviors treated as if they were building blocks that are what they are in themselves. But if Dewey was right, then this is not the way to proceed with investigations because perceptions and so forth can only be understood by recognizing the role they play in what people are doing, which comes first.

The second reason is that theorists and researchers are part of the world of shared practices themselves, always already engaged in doing things. Therefore, what they observe will always depend on how they are already "looking." At one level, the way they "look" will be based on specific practices for inquiry in their area of the field. Those specific practices in turn will be embedded in more basic practices based on being a member of their culture at large who, of course, is embodied. Rather than trying to circumvent being a participant in practices, which ultimately is impossible, we will get further - and succeed at refining, and even in some ways altering, our understanding of the things people do - by proceeding in ways that start with our practical familiarity with the practices of interest.

One other implication of Dewey's position regarding the primacy of practices relates closely to the preceding point. Researchers in psychology should investigate phenomena of interest in a context-sensitive manner. This includes relating a person's cognitions or individual behaviors to events that are occurring in a given situation. It also includes appreciating the roles played by those cognitions, behaviors and events in what the person is trying to do, that is, the practical activity in which he or she is engaged. One reason for this is that, as we said in the prior point, it will not get us that far if we treat cognitions, behaviors, and so forth as starting points, as if they were building blocks that are what they are in themselves. Another reason is a different, positive way of looking at the same idea. If we consider an individual behavior or perception, for example, in terms of the role it plays in what a person is doing, we can enhance our understanding of that part of the practical activity in which the person is engaged.

\footnotetext{
${ }^{1}$ Dreyfus (1999) offered a very convincing argument against social constructionism in a critique of Searle's (1995) book, “The Construction of Social Reality." His points are compatible with the notion of the primacy of practices, although Dreyfus is not thought of as a pragmatist.
} 


\section{IDEAS FOR FUTURE RESEARCH}

We now turn to considering whether and, if so, how researchers could follow-up our comparison of IRT and therapy based on Interpersonal Defense Theory. Some of the points we will make follow from our preceding remarks about pragmatism. Also, in a number of places, we indicate where our suggestions highlight and/or build upon how Messer and his collaborators compared two other approaches in the program of research described in Messer's commentary (Collins \& Messer, 1991; Holland et al., 1998; Messer et al., 1992; Tishby \& Messer, 1995). In addition, in this part of the paper, we also point out suggestions that we believe have relevance for therapy research more generally.

Messer's question about whether it is possible to compare therapy based on IRT and Interpersonal Defense Theory merits consideration. In the second paper in this set of articles, we pointed out one reason in particular that suggests it might not be possible to compare them. We noted that although MW, on the one hand, and $\mathrm{KC}$, on the other, had access to virtually the same set of materials from Sharon's case, the evidence each of us actually focused on was quite different. Our subsequent papers from each of our two perspectives make this difference clear. In the second paper, we explained that this feature of our project is not unique in the sense that whenever case formulations are used in research or practice, they direct attention to certain aspects of a case and not others. This point notwithstanding, it seems reasonable to wonder what to make of efforts that lead us to only consider some kinds of evidence.

If we take a wider view about theorizing and conducting research, this question about how evidence is considered appears once again. For example, Max Weber (1949) argued in a well-known chapter that throughout the social sciences it is impossible to arrive at conclusions that are objectively true because it is simply impossible to consider the infinite number of different features of phenomena of interest. Weber maintained that what theorists and researchers do instead is focus on the small subset that they view as important given their values.

In fact, Weber's view is not remarkable at all. One of the main things that theories do is specify what aspects of things are relevant. If we go back to the billiard ball example, Newtonian mechanics tell us that when a stationary ball is struck by a moving ball, it will move in a particular direction at a certain speed. The theory directs us to consider the trajectory, speed, and mass of the striking ball and the mass of the ball that gets struck, and not, for example, the colors of the balls.

At first glance, Dewey's idea of the primacy of practices may appear to make this problem even more far-reaching. According to that idea, theories per se (the explicit ideas that one writes down on a piece of paper) are embedded in the concretely meaningful practices of the relevant field (e.g., what kinds of theory-based research are likely to get funded at a particular point in time) and those disciplinary practices, in turn, are embedded in the practices of our everyday lives. According to the primacy of practices, the facts (e.g., the mass of one of the billiard balls) are not brute evidence. They are not what they are in themselves. Rather, what they 
are cannot be separated from the roles they play in the practical activities in which we are already engaged.

Nevertheless, pragmatism also tells us that we can resolve the problem. The key is the possibility of certain kinds of agreements. Perhaps the main type of agreements of this sort concerns what we might call "third points." If Interpersonal Defense Theory focuses on X, Y, and $\mathrm{Z}$ items of evidence and IRT focuses on $\mathrm{A}, \mathrm{B}$, and $\mathrm{C}$ items of evidence, can the proponents of both those approaches agree that $\mathrm{M}, \mathrm{N}$, and $\mathrm{O}$ items of evidence are relevant in evaluating both approaches to therapy?

Pragmatism suggests that there is every reason to believe that it will be possible to find the necessary third points precisely because theories are not hermetically sealed ideal structures. That is, each theory is not completely a fully self-contained set of propositions. As we noted above, theories are embedded in much broader disciplinary practices and those practices, in turn, are embedded in everyday practices. The upshot is that most often advocates of two different theories share in common familiarity with many relevant practices that can serve as the basis for agreeing on third points (for some examples, see the next section).

More generally, even when researchers are not proponents of a viewpoint under investigation, they can usually evaluate claims made about a single case formulation approach to psychotherapy or other claims about therapy based on particular theories because of this shared familiarity with broader, but still relevant practices. In these cases, they are likely to employ second "points," that is, kinds of evidence that are not directly linked to the position under investigation, but that are relevant to it, in addition to the kinds of evidence that are directly linked to that position (i.e., first points). Here again, shared familiarity with broader practices makes possible this way of proceeding.

We should explicitly state that the necessary agreements about second or third points are not based on brute facts that are what they are in themselves; they are points of agreement that are based on shared familiarity with practical activities. Not surprisingly, from the perspective of pragmatism, we cannot resolve the problem at hand by resorting to correspondence theory.

Pragmatism also offers one more helpful idea. Another way practices make it possible to resolve disagreements between theories is that we are familiar with practices for doing just that. Some of those practices are specific to particular disciplines. Others are part of our everyday lives.

\section{Outcome Measures As “Third Points"}

Outcome measures probably are the most important kind of third point for comparing two perspectives on therapy that employ case formulations. For the present project, we agreed that the battery of measures described in the second paper in this set of articles provided an acceptable basis for the assessment that the outcome of Sharon's case was poor. For example, although the Inventory of Interpersonal Problems (Horowitz, Alden, Wiggins, \& Pincus, 2000), which was one of the measures in the battery, does not assess interpersonal difficulties in a way 
that closely follows along the lines of either of our theoretical perspectives in general or our formulations of Sharon's problems in particular, we could agree that the measure captures changes relevant to each of our viewpoints in broad strokes. For similar reasons, both of us were willing to treat Sharon's scores on the other measures in the battery as third points that would serve as the criteria for investigating the predictive validity of our respective case formulations. Each of us did that by examining the clinical material to see whether the evidence that seemed important from our own perspective provided a way to explain the poor outcome.

In our opinion, future research comparing our approaches might well employ a similar method for assessing outcome. To be sure, it would not be so easy to arrive at agreement about assessing outcome in studies comparing some other pairs of therapy approaches, for example, an approach aimed at improvements in symptoms and another aimed at promoting changes in personality structure. From the vantage point of pragmatism, the difficulty at arriving at agreed upon third points in this hypothetical situation results from the complex array of ways that matters related to "the good life" run though our everyday practical activities.

Nevertheless, there is a sense in which agreement is possible even when it comes to these difficult comparisons. Specifically, researchers could include outcome measures related to both sets of concerns. As we see it, proceeding in this manner is possible because all of the investigators involved would understand what was being assessed by both sets of measures and why a proponent of the other approach might believe that their measures were assessing something important. Those investigators and other psychologists reading reports of their research would be able to decide how they wanted to proceed whatever pattern of findings was found concerning the two sets of outcome measures. We would say that, here again, familiarity with shared practices provides the basis for "agreements" of this limited sort.

\section{Other Suggestions For Future Research}

What about other features of possible future research besides outcome? Not surprisingly, we believe that any further research comparing Interpersonal Defense Theory and IRT should take a case formulation approach. That was a basic feature of the present project, and it is key to both of our perspectives. It also was the approach Messer and his collaborators took in their research. However, the case formulations for both theoretical orientations they studied, Mt Zion and Rutgers, followed the Mt Zion scheme regarding what the components of the formulations would be (e.g., both sets of formulations included a component about patient "tests"). In our project, the components of formulations were different for our two approaches because our theoretical perspectives identify very different aspects of a case as important. Most likely, this would also be the case for future comparisons of most other pairs of therapy approaches.

We believe that case formulation-based research has great potential for contributing to our understanding of therapy. In fact, Persons (1991) argued that this approach might well help the field unravel the puzzle of the apparent equivalence of outcomes found in many studies comparing two or more therapy approaches. Typically, those studies randomly assign patients with a particular DSM diagnosis to manualized versions of the treatments under investigation. 
However, there is considerable variability between patients who share the same diagnosis. The case formulation approach to research makes it possible to assess how well a particular treatment orientation has been applied to each individual patient. We believe that taking a case formulation approach can also make contributions to investigations of many other issues about psychotherapy in addition to comparing the effectiveness of different therapies.

Another question about how to conduct future research comparing therapy approaches concerns what treatment condition or conditions to include in those studies. Messer and his collaborators employed the strategy of studying one case treated by the Rutgers object-relations approach and another case treated by the Mt Zion control-mastery approach. This design may be the best way to proceed in many future studies.

For the present project, however, we proceeded in a different way, studying a case treated by an approach that was based on a different approach from both Interpersonal Defense Theory and IRT. Given that our study included only one case, this was a better way to proceed than to use a case conducted based on one of our theoretical perspectives. It is possible that using a therapy approach that differs from two therapy perspectives under investigation might also be helpful for other studies that include multiple cases. One positive feature of this design is that it controls for person-of-the-therapist effects.

Another point to consider for future studies comparing our two approaches or any two approaches to therapy is the importance of examining therapist behaviors. In fact, we believe that all such studies should be process-outcome investigations. Most research comparing approaches to therapy goes as far as assessing adherence of the therapists in each treatment condition to a manual for that approach. In most cases, adherence measures simply assess whether a therapist employs the types of interventions called for by the therapy approach under investigation. When studies are based on case formulations, a different and closer examination of therapist behavior is called for because it must be assessed in terms of whether it conforms to what is called for by the therapy approach being studied given the formulation of each case.

For example, based on Interpersonal Defense Theory, future studies comparing that approach to IRT might assess whether therapists intervened in ways that could help patients arrive at insights about their central interpersonal wish in accord with formulations identifying what that wish was for each case. Based on IRT, they could assess whether therapists intervened in ways that might help patients recognize similarities between "Red" patterns from their childhoods and their current interpersonal behavior, as the Red pattern for each patient was identified in a case formulation.

The research by Messer at al. proceeded in just this way. For both cases studied, they assessed whether the therapist's behaviors conformed to the case formulation for that case based on the object relations perspective and whether the therapist's behaviors conformed to a formulation based on control mastery theory.

Two other points about therapists' behaviors merit consideration. As we noted earlier in this paper, pragmatism suggests that it is important to investigate phenomena of interest in a 
context-sensitive manner. This should be kept in mind when developing ways to measure the fit between a therapist's behaviors and a case formulation. Interestingly, this was a key consideration in the research by Messer and his collaborators (see especially, Messer et al., 1992).

The other point is that depending on the guiding theory, it may be important to consider if therapist behaviors conform to the formulation of a case on the level of therapy relationship processes as well as in terms of content. For example, according to Interpersonal Defense Theory, it is at least as important to consider whether a therapist's response realizes a patient's interpersonal wish in the patient-therapist relationship in accordance with the case formulation as it is to determine whether the content of that therapist bid accurately identifies the patient's wished-for kind of interpersonal response. As we understand the research by Messer and his coauthors, it focused solely on content, but that might have been entirely on target given the two theoretical perspectives under investigation.

\section{Alternative Approach To Examining Validity}

We should offer some brief remarks about an alternative to comparing therapy approaches by taking outcome measures as the criteria of predictive validity. It is also possible to compare the validity of two case formulation approaches to therapy by examining whether therapist behaviors in accord with each approach given the formulation of a case based on that approach lead to proximal changes predicted by the respective theoretical perspectives.

Messer et al. took this approach. They investigated associations between their two assessments of therapist behavior (one based on the Mt Zion formulations, the other based on the Rutgers formulations) and "patient progress" assessed at a number of points during treatment. As Messer explained in his commentary, the patient progress measure assessed such things as whether the patient explored significant material, developed insight, and focused on self, and the patient's emotional experiencing.

We see this as a promising way to proceed, although in future research comparing Interpersonal Defense Theory and IRT, or in future studies comparing other pairs of approaches, we think that it would be most helpful to study proximal changes or changes assessed at termination specific to each of the approaches under investigation. Research of this sort can be highly informative about mechanisms of change. For example, in a study comparing Interpersonal Defense Theory and IRT, (a) to explore the validity of a tenet of Interpersonal Defense Theory, an investigator could examine whether therapist bids that are countertransferential (as that concept is understood in Interpersonal Defense Theory and according to the case formulation for each case) promote defensive, noncoordinating responses by patients, and (b) as a way of exploring the validity of a claim of IRT, a researcher could examine whether therapist interventions of the kind called for by that therapeutic perspective given the formulation of each case lead to changes in patients' commitment to the Gift of Love hypothesis. An interesting way to augment such studies would be to investigate whether the interventions suggested by one theoretical perspective (e.g., Interpersonal Defense Theory) lead 
to the theory specific changes identified as important by the other theoretical perspective (e.g., IRT) and vice versa.

Putting aside this very last suggestion, we should also point out that it is possible to combine studies that examine whether the interventions called for by a given perspective lead to changes specific to that perspective with assessments of outcome at termination that are general, rather than theory specific, in nature. Such investigations could see whether the therapist behaviors of interest promote the theory-specific changes, and whether those changes in turn predict overall improvement.

\section{Small N Studies}

Most of our suggestions could be implemented in quantitative investigations of a very small number of cases as well as in quantitative research involving larger groups of cases. The research by Messer and his collaborators provides an excellent illustration of the possibilities for conducting informative small $n$ quantitative research comparing approaches to therapy.

One way to build on that research would be to use paired comparisons to include assessments of outcome at the end of treatment as predictive validity criteria. Designs of this kind are based on pairs of cases treated by the same therapist, with one case in each pair assessed as having had a poor outcome and the other case assessed as having had a good outcome. Useful studies could be based on one paired comparison for which the therapy approach guided by Interpersonal Defense Theory and one other paired comparison for which the approach was IRT (or any two theoretical perspectives on therapy). As in the research by Messer et al., formulations for all four cases would be arrived at based on both theoretical perspectives by two independent sets of judges (one set for each perspective). Other independent judges would examine therapist behaviors in each case to determine whether they conformed to specific features of the formulations for each case.

We also believe that future qualitative case studies of the kind we conducted can be useful as well. Such studies could be based on a single case treated by a therapist guided by a third therapy approach, as was the case in the present project, or one paired comparison treated by a third approach, or two paired comparisons with each pair treated by a therapist guided by one of the theoretical perspectives being compared.

We believe that qualitative studies have certain advantages over quantitative investigations. For one thing, they provide an opportunity to learn a great deal about what hypothesized processes look like concretely. As such, they can offer very helpful guidance about how to operationalize constructs of interest for subsequent quantitative studies. In our opinion, they also have advantages in their own right. One point here is that they facilitate moving from research to practice. Another point is that they can be pursued as theory building case studies. As Stiles $(2007,2009)$ explained, in comparison to most quantitative studies, theory building case studies have the advantage that a researcher can investigate the multiple tenets of theory simultaneously. When close qualitative examination of a case indicates that observations 
simultaneously conform to those multiple tenets, this provides a special kind of incremental support for the validity of theoretical perspectives.

A practical consideration also points to the usefulness of small $n$ quantitative and qualitative studies. When comparing therapy approaches, it is possible that an approach that is especially effective for one kind of case might not be very helpful for another kind of case. For example, given the fact that for the most part studies of IRT have involved patients with more serious problems than has been true for studies investigating Interpersonal Defense Theory, it is possible that the relative advantages of one of these approaches over the other might depend on severity of disorder in general, or perhaps, severity of personality disorders in particular (borderline personality disorder versus avoidant personality disorder). Small $n$ studies have the practical advantage that investigators can more readily (i.e., quickly) learn something about the many possible factors of this type that might influence the relative merits of one approach over another, although, of course, we would not be able to place as much confidence in the results of such small $n$ studies as compared to findings from otherwise parallel large $n$ studies. ${ }^{2}$

\section{CONCLUDING COMMENTS}

We wish to express our thanks once again to Stanley Messer for the questions he posed in his commentary about whether it is possible to compare different approaches to therapy and, if it is, how researchers should go about studies of that kind; for linking his comments to philosophical theories about truth, including, in particular, the pragmatic theory of truth; and for offering research he and his collaborators conducted as a possible example for how to proceed, which we believe is an excellent example to consider.

In this reply to his commentary, we have tried to build on Messer's remarks by discussing Dewey's classic critique of the reflex arc concept as a way of pointing out other features of the philosophical perspective of pragmatism. Those other features speak in helpful ways to why different approaches to psychotherapy are not incommensurate. We have also offered a number of suggestions about future research comparing psychotherapy therapy based on Interpersonal Defense Theory and IRT, or any two therapy approaches. At many points, our suggestions followed along the lines of Messer's research.

To be sure, the kind of studies we have recommended are not easy to conduct. The main reason for this is that they are based on case formulations. However, studies of this kind have many advantages over most efforts to compare treatment approaches. In particular, they provide individualized conceptualizations of patient's problems based on a given theoretical perspective, and this then makes possible individualized conceptualizations of the treatment implications of

\footnotetext{
${ }^{2}$ Small $n$ studies have another practical advantage due to the fact that the kinds of research we are suggesting are much more labor and resource intensive than standard RCTs. Unfortunately, funding priorities encourage studies on whether and for whom therapies work, rather than on how they work. To be sure, the best solution would be that investigators would proceed with the small $n$ studies we have suggested, and that funding priorities would change so that there would also be support for large $n$ studies that take a case formulation approach and investigate mechanisms of change.
} 
the theoretical perspective for each case. As we pointed out, one upshot of this way of proceeding is that studies comparing therapy approaches have to be conducted as processoutcome studies, not just studies comparing outcomes of two kinds of therapies with each approach simply studied as a treatment package. We also pointed out how case formulationbased studies can be conducted in ways that are highly informative about mechanisms of change. Although it is more challenging to conduct case formulation-based research, we believe that proceeding in this way enhances the validity and clinical usefulness of efforts to compare approaches to therapy.

\section{REFERENCES}

Collins, W. D., \& Messer, S. B. (1991). Extending the Plan Formulation Method to an object relations perspective: Reliability, stability and adaptability. Psychological Assessment: A Journal of Consulting and Clinical Psychology, 3, 75-81.

Critchfield, K.L., Dobner-Pereira, J., \& Stucker, E. (2021a). The case of Sharon considered from the vantage point of Interpersonal Reconstructive Therapy. Pragmatic Case Studies in Psychotherapy, 17 (1), Article 4, 42-62. Available: http://pcsp.libraries.rutgers.edu/

Critchfield, K.L., Dobner-Pereira, J., \& Stucker, E. (2021b). Interpersonal wishes and fears with regard to internalized attachment figures: Differing focus of two case formulation methods that use SASB. Pragmatic Case Studies in Psychotherapy, 17 (1), Article 6, 85103. Available: http://pcsp.libraries.rutgers.edu/

Dewey, J. (1896). The reflex arc concept in psychology. Psychological Review, 3, 357-370.

Dreyfus, H. L. (1999). The primacy of phenomenology over logical analysis. Philosophical Topics, 27, 3-24.

Holland, S. J., Roberts, N.E., \& Messer, S. B. (1998). The reliability and validity of the Rutgers Psychotherapy Progress Scale. Psychotherapy Research, 8, 104-110.

Horowitz, L. M., Alden, L. E., Wiggins, J. S., \& Pincus, A. L. (2000). Inventory of Interpersonal Problems. New York, NY: The Psychological Corporation.

Messer, S.B. (2021). How do we decide which of two case formulations is correct? Commentary on Westerman and Critchfield et al. Pragmatic Case Studies in Psychotherapy, 17 (1), Article 7, 104-108. Available: http://pcsp.libraries.rutgers.edu/

Messer, S. B., Tishby O., \& Spillman, A. (1992). Taking context seriously in psychotherapy research: Relating therapist interventions to patient progress in brief psychodynamic therapy. Journal of Consulting and Clinical Psychology, 60, 678-688.

Persons, J. B. (1991). Psychotherapy outcome studies do not accurately represent current models of psychotherapy. American Psychologist, 46, 99-106.

Searle, J. R. (1995). The construction of social reality. New York: Free Press.

Spence. (1982). Narrative truth and historical truth. New York: Norton.

Stiles, W. B. (2007). Theory-building case studies of counselling and psychotherapy. Counselling and Psychotherapy Research, 7, 122-127.

Stiles, W. B. (2009). Logical operations in theory-building case studies. Pragmatic Case Studies in Psychotherapy, 5, 9-22. Retrieved from jrul.libraries.rutgers.edu/index.php/pcsp/ article/view/973/2384

Tishby, O., \& Messer, S. B. (1995). The relationship between plan compatibility of therapist 
interventions and patient progress: A comparison of two plan formulations. Psychotherapy Research, 5, 76-88.

Weber, M ( 1949). "Objectivity" in the social sciences and social policy. In E.A. Shils \& H.A.Finch (Trans. and Eds.), The methodology of the social sciences (pp. 49-112). New York: The Free Press.

Westerman, M.A. (2021a). The case of Sharon considered from the vantage point of Interpersonal Defense Theory. Pragmatic Case Studies in Psychotherapy, 17 (1), Article 3, 19-41. Available: http://pcsp.libraries.rutgers.edu/

Westerman, M.A. (2021b). Comparing Interpersonal Defense Theory and Interpersonal Reconstructive Therapy and their views of Sharon's case. Pragmatic Case Studies in Psychotherapy, 17 (1), Article 5, 63-84. Available: http://pcsp.libraries.rutgers.edu/ 